To the Editors:

\title{
Practices related to sharps disposal among ambulatory patients with diabetes on insulin therapy
}

\author{
K R Atukorala ${ }^{1}$, R D N Sumanasekera ${ }^{2}, \mathrm{~K} \mathrm{H}$ Wickramasinghe $^{3}$, S I Wickramasinghe ${ }^{4}$ \\ Ceylon Medical Journal 2016; 61: $91 \quad$ DOI: http://doi.org/10.4038/cmj.v61i2.8298
}

Diabetes mellitus has grown into epidemic proportions in Sri Lanka [1, 2]. Management of diabetes requires regular blood sugar assessment, oral medications and insulin injections. These procedures generate sharps within the household and improper disposal has the potential to cause public health problems such as personal injury and propagation of blood borne infections via needle stick injuries.

Sharps disposal practices within hospital settings in both public and private sector are regulated by the government. But public health guidelines and public services for home based sharps disposal are currently unavailable. Considering the above, we conducted a study to determine how patients dispose of the sharps they use at home. It was a descriptive cross-sectional study among ambulatory patients with diabetes using insulin, who were being followed up at the North Colombo Teaching Hospital (CNTH). Participants were randomly selected and informed consent was obtained.

Data were collected on sharps disposal practices using an interviewer-administered questionnaire by trained research assistants. The questions explored insulin administration practices, equipment used, frequency of needle use, disposal of insulin syringes, pens and lancets, and knowledge regarding spread of diseases by sharing needles. Approval was obtained from the Ethics Review Committee, Faculty of Medicine, University of Kelaniya.

Data were collected from 158 consenting patients. Their age ranged between 21 to 90 years (mean 60 years). The mean duration of diabetes was 7.4 years. Average duration of insulin administration was 3.2 years and $83 \%$ (131/158) had used insulin injections for more than one year. Eighty four percent (132/158) of patients required more than two doses of insulin a day. Only 5/158 (3\%) patients used the insulin pen and the majority (153/158) used syringes. Majority 150/153 (98\%) recapped and reused the same needle repeatedly. More than $50 \%$ of patients reported that they used the same needle up to six times (over 3 days). Seventy four percent of the participants (117/158) cleaned the injection site before administering the injection. However, only 10/158 (6\%) patients regularly self-monitored blood sugar levels. Forty six percent (73/158) of the patients got help from others when injecting insulin and also in disposing of sharps.

Commonly generated sharps in the household were disposed into a common household garbage bin by $68 \%$ (107/158) of patients. The other methods used to dispose sharps were sharps container, toilet pit, common public garbage dump and indiscriminate methods. Some patients had tried to bury or burn sharps. But needles and lancets cannot be incinerated by domestic fires. Also toxic fumes may be released in burning plastic syringes in home gardens.

The Sri Lankan healthcare delivery system may lack the financial resources for systems used in developed countries, such as sharps "mail back" programmes or at providing home needle destruction devices [3]. Simple solutions such as using a 'hard plastic' bottle to collect sharps at home and later discarding at a local hospital or a designated counter could be a solution. Commercial providers should also be encouraged to provide safe sharps disposal options in public places.

\section{Conflicts of interests}

There are no conflicts of interest.

\section{References}

1. Katulanda P, Sheriff MH, Matthews DR. The diabetes epidemic in Sri Lanka - a growing problem. The Ceylon Med J 2006; 51: 26-8.

2. Katulanda P, Constantine GR, Mahesh JG, et al. Prevalence and projections of diabetes and pre-diabetes in adults in Sri Lanka - Sri Lanka Diabetes, Cardiovascular Study (SLDCS). Diabet Med 2008; 25: 1062-9.

3. BD diabetes learning centre. Safe needle disposal: United States. 2015: http://www.bd.co3. BD diabetes learning centre. Safe needle disposal: United States. 2015: http:// www.bd.c

${ }^{1}$ Department of Physiology, Faculty of Medical Sciences, University of Sri Jayewardenepura, ${ }^{2}$ Department of Family Medicine, Faculty of Medicine, University of Kelaniya, ${ }^{3}$ National Eye Hospital, Colombo and ${ }^{4}$ Ministry of Health, Colombo, Sri Lanka.

Correspondence: RDNS, e-mail: <rndeepama@gmail.com>. Received 1 October and revised version accepted 30 November 2015.

This is an open-access article distributed under the terms of the Creative Commons Attribution License, which permits unrestricted use, distribution, and reproduction in any medium, provided the original author and source are credited. 